\title{
Nanostructured Optoelectronics: Materials and Devices
}

\author{
Hieu P. T. Nguyen, ${ }^{1}$ Shamsul Arafin, ${ }^{2}$ J. Piao, ${ }^{3}$ and Tran Viet Cuong ${ }^{4}$ \\ ${ }^{1}$ Department of Electrical and Computer Engineering, New Jersey Institute of Technology, Newark, NJ 07102, USA \\ ${ }^{2}$ Department of Electrical and Computer Engineering, University of California, Santa Barbara, CA 93106, USA \\ ${ }^{3}$ Epitaxial Laboratory, Inc., Dix Hills, NY 11746, USA \\ ${ }^{4}$ Department of Solid State Physics, University of Science, Vietnam National University, Ho Chi Minh City, Vietnam
}

Correspondence should be addressed to Hieu P. T. Nguyen; hieu.p.nguyen@njit.edu

Received 11 July 2016; Accepted 11 July 2016

Copyright (C) 2016 Hieu P. T. Nguyen et al. This is an open access article distributed under the Creative Commons Attribution License, which permits unrestricted use, distribution, and reproduction in any medium, provided the original work is properly cited.

The use of nanostructure materials for optoelectronic devices, including light-emitting diodes (LEDs), laser diodes, photodetectors, and solar cells, has recently attracted considerable attention due to their unique geometry. Nanostructures in small dimensions can be perfectly integrated into a variety of technological platforms, offering novel physical and chemical properties for the high performance optoelectronic devices. The exploitation of new nanostructures and their optical and electrical properties is necessary for their emerging practical device applications.

This special issue contains six papers, presenting some recent advances in the theoretical calculation, synthesis, characterization, and application of such novel nanostructures.

Recently, natural dyes have been widely studied as potential sensitizers for dye-sensitized solar cells (DSSCs) due to their cost efficiency, nontoxicity, and complete biodegradation. In "Photoactive Layer of DSSCs Based on Natural Dyes: A Study of Experiment and Theory," Y. Li et al. investigated, both theoretically and experimentally, three natural dyes for DSSCs which were extracted from natural plants, including Forsythia suspensa, Herba Violae, and Corn leaf. The authors reported that such natural dyes exhibit wide absorption region which covers almost the whole visible spectrum. The highest photoelectronic conversion efficiency for these natural dyes was recorded to be $0.96 \%$ with open circuit voltage of $0.66 \mathrm{~V}$ and short circuit current density of $1.97 \mathrm{mAcm}^{-2}$ which is promising for future biophotovoltaics applications.

Among these novel nanostructures, recent rapid advances in research involving two-dimensional (2D) layered nanomaterials and nanoplasmonics could pave the way for developing next-generation optoelectronic and photonic devices. Furthermore, the use of such $2 \mathrm{D}$ materials as a buffer layer for the growth of light-emitting III-V compound semiconductors by the so-called van der Waals epitaxy method has opened up a new route of heteroepitaxy, mitigating a lot of growthrelated technological challenges. As an effort, this special issue features a theoretical paper relating to emerging $2 \mathrm{D}$ materials. X. Hu and F. Meng in "First-Principle Study on the Interaction between Fe and Trivacancy in Graphene" have reported the interaction between iron metal and monolayer graphene. In fact, this study has described a detailed investigation on the structural and electronic properties of graphene with vacancies as well as the advantages offered by having these vacancies on the graphene surface. These results certainly provide insights to engineer the electrical properties of graphene through defect addition and manipulation, being useful for industrial semiconductor applications such as the photocatalytic technology and graphene-based electronics.

In another paper, W. Sukkabot theoretically studied the impact of structure shapes on the electron-hole exchange interaction in core/shell nanostructure semiconductors. The study focused on the electron-hole exchange interaction in the morphological transformation of $\mathrm{CdSe} / \mathrm{ZnS}$ core/shell nanodisk to $\mathrm{CdSe} / \mathrm{ZnS}$ core/shell nanorod using atomistic tight-binding theory and a configuration interaction description. The aspect ratios were successfully used to study the structural and optical properties of such nanostructures. The single-particle and excitonic gaps are believed to be decreased by changing from disk to rod shapes. The authors concluded that light hole is suggested to be used for quantum information instead of a heavy hole. This study contributes 
important information to the design of high performance II-VI semiconductor nanocrystals for optoelectronic applications.

Y. J. Park et al. reported their study on the enhanced light extraction efficiency of LEDs by employing two $\mathrm{ZnO}$ nanostructures. The experiments were performed on two types of nanostructures including one-dimensional nanorods and two-dimensional nanosheets which were grown directly on top of LEDs. The formation of surface texturing on LEDs with $\mathrm{ZnO}$ nanorods offers increased escape cone and surface scattering, resulting in the enhancement of light output power of $30 \%$ compared to conventional LEDs without using $\mathrm{ZnO}$ nanostructures. However, due to the increased internal reflection and light absorption in $\mathrm{ZnO}$ nanosheets, LEDs using nanosheet structures have lower light output efficiency compared to the conventional one. The employment of $\mathrm{ZnO}$ nanorods shows promising approach for the enhanced output power of LEDs. More importantly, LED devices are not severely suffering from degradation of electrical properties by using $\mathrm{ZnO}$ nanorods.

In the paper "A Method to Control Dynamic Errors of the Stylus-Based Probing System for the Surface Form Measurement of Microstructures," H. Fang et al. proposed a simple and cost-effective method to control dynamic errors of the stylus-based probing system on measuring the surface form of microstructures. The dynamic errors were numerically simulated and suggested that the scanning speed and initial position of the measured specimen directly affect the dynamic errors. The authors proposed a solution to enhance the form measurement accuracy of microstructures by using kinematical models to predict the influence of the measurement setup on dynamic performance. The dynamic errors, therefore, can be controlled by properly choosing the optimal scanning speed and the initial position of the measured samples.

V. R. Balaji et al. presented a novel design of twelvechannel Dense Wavelength Division Multiplexing (DWDM) demultiplexer using the two-dimensional photonic crystal (2D PC) square resonant cavity. The rod radius and wavelength were optimized by linear regression analysis. The authors claimed that their DWDM exhibits high accuracy of $95 \%$ with an average quality factor close to 8000 . Importantly, the proposed PC based demultiplexer has small figure size and is perfectly applied in integrated optics.

\section{Acknowledgments}

The Guest Editors would like to thank all authors for their contributions to this special issue. They would also like to acknowledge reviewers of this special issue for their time and comments.

Hieu P. T. Nguyen

Shamsul Arafin

J. Piao

Tran Viet Cuong 

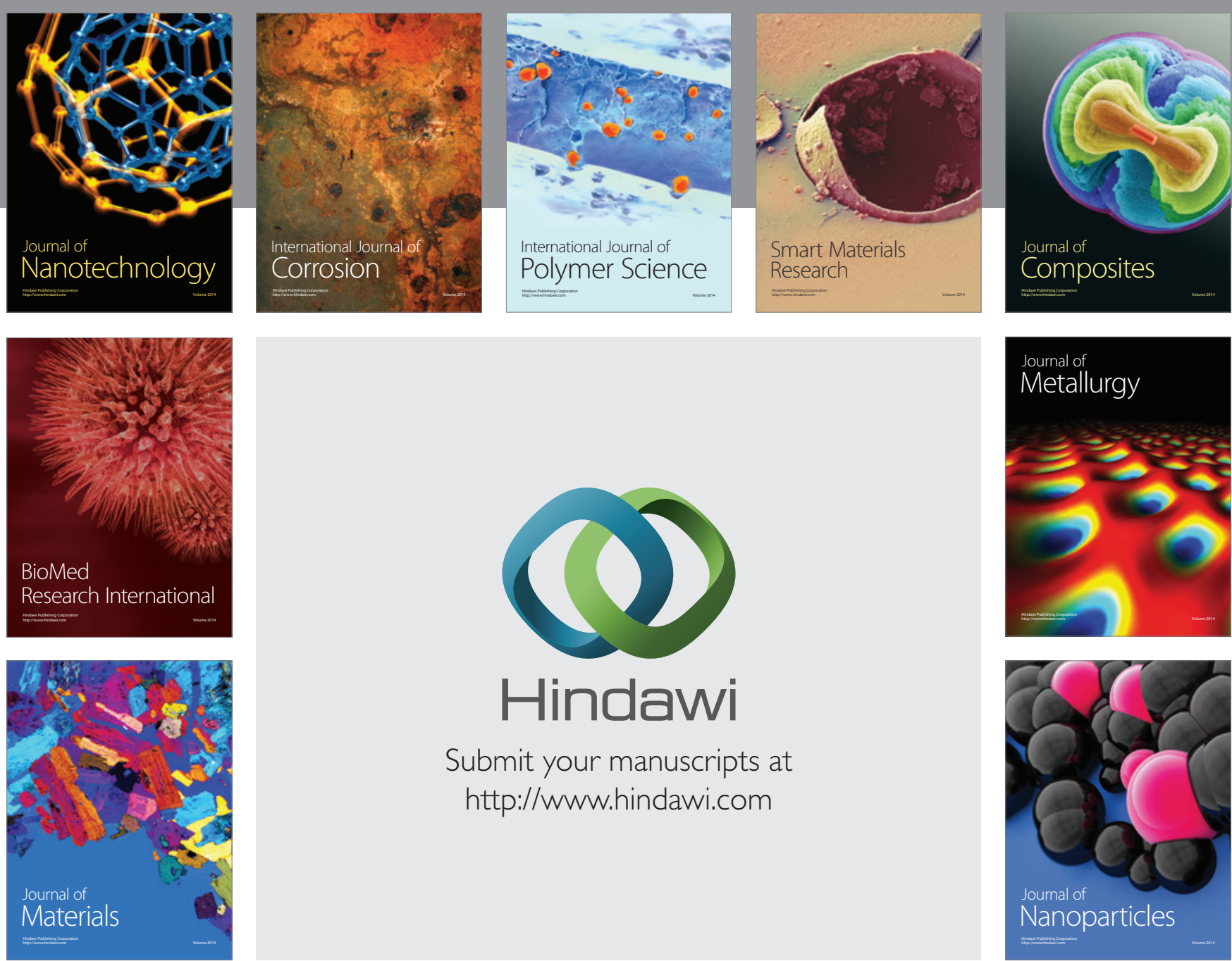

\section{Hindawi}

Submit your manuscripts at

http://www.hindawi.com

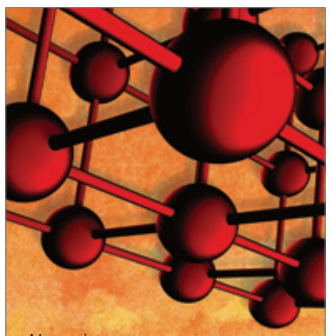

Materials Science and Engineering
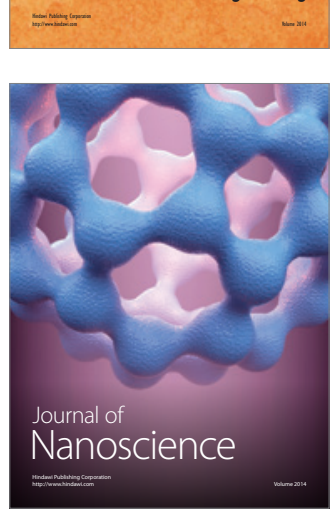
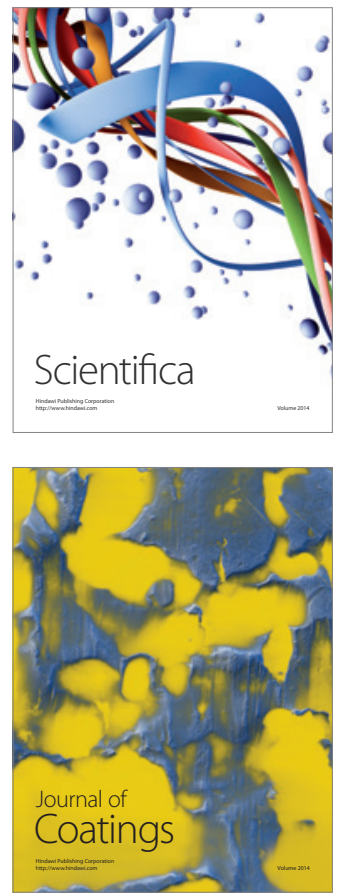
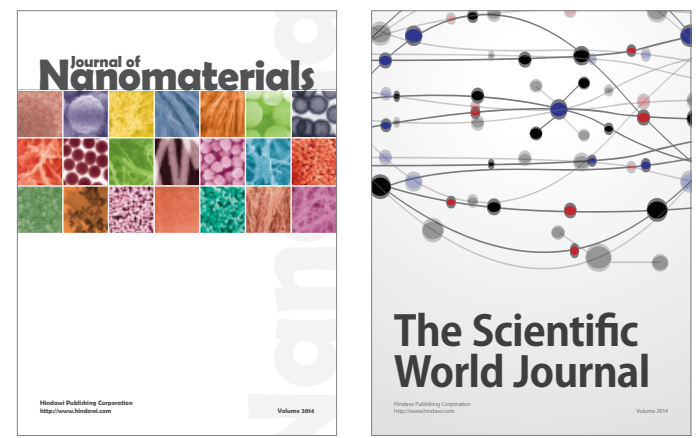

The Scientific World Journal
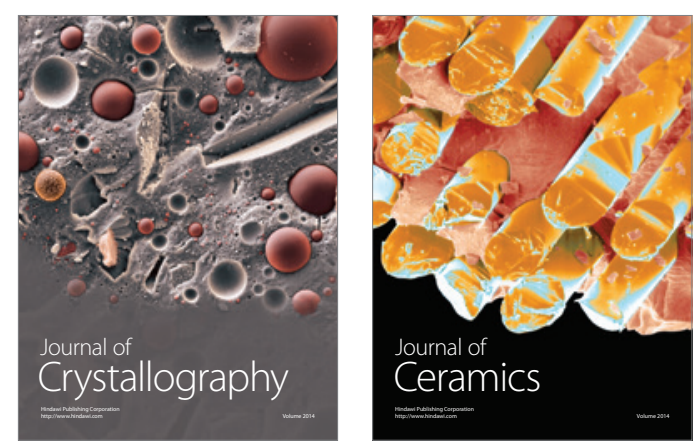
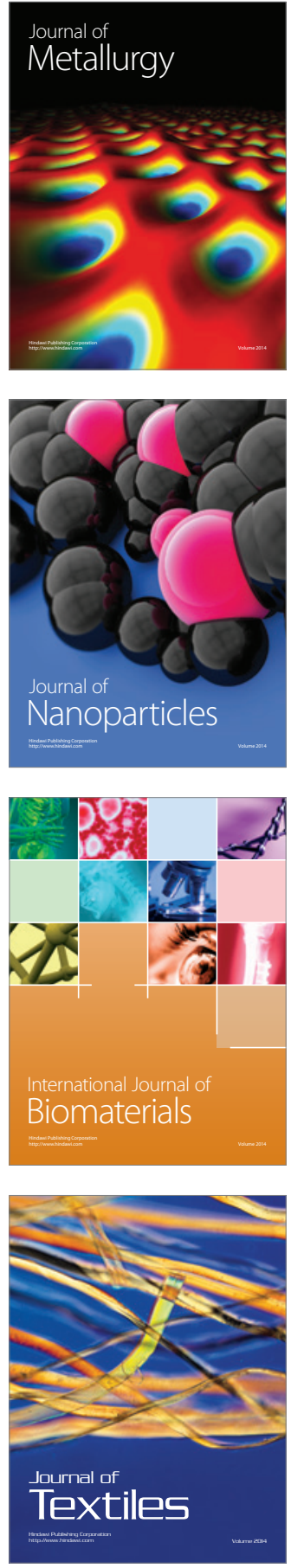